\title{
Differentiation between perceived effort and discomfort during resistance training in older adults: Reliability of trainee ratings of effort and discomfort, and reliability and validity of trainer ratings of trainee effort
}

\author{
James Steele, James Fisher, Stephen McKinnon, Pat McKinnon
}

\begin{abstract}
Objectives: Rating of perceived exertion scales are commonly used in resistance training (RT) though most suffer from conflation of perceptions of both effort and discomfort by participants. The aim of this study was to examine reliability of trainee ratings of perceived effort (RPE-E) and discomfort (RPE-D) using two novel scales in addition to reliability and validity of trainer RPE-E.

Design: Participants underwent 3 RT trials over a period of three weeks.

Methods: Seventeen participants (males $n=6$, females $n=11$, age $63 \pm 16$ years) completed 5 RT exercises for a single set using a load permitting a self-determined 6 repetition maximum (meaning they determined inability to complete further repetitions if attempted i.e. they predicted momentary failure on the next repetition). Trainers completed their rating of RPE-E, followed by participants reporting of RPE-E and RPE-D immediately after completion of the exercises. Spearman's correlations examined the relationship between RPE-E and RPE-D. Reliability was examined as standard error of measurement (SEM) calculated for each outcome across the 3 trials (intra-rater), in addition to agreement between trainers (inter-rater), and agreement between trainer and trainee RPE-E.

Results: Correlations between RPE-E and RPE-D were significant but weak $(r=.373$ to $0.492 ; p<0.01)$. Intra-rater SEMs for trainee RPE-E ranged from 0.64 to 0.85 , trainee RPE-D ranged from 0.60 to 1.00 , and trainer RPE-E ranged from 0.56 to 0.71 . Inter-rater SEMs for trainer RPE-E ranged 0.25 to 0.66. SEMs for agreement between trainer and trainee RPE-E ranged from 1.03 to 1.25 .

Conclusions: Results suggest participants were able to differentiate RPE-E and RPE-D and that the reliability for both trainee measures of RPE-E and RPE-D, in addition to trainer RPE-E is acceptable. Further, trainer RPE-E appeared to have acceptable validity compared to trainee RPE-E. These scales might be adopted in research examining the dose-response nature of effort upon RT outcomes and trainers might use them to inform programming.
\end{abstract}

(Journal of Trainology 2017;5:1-8)

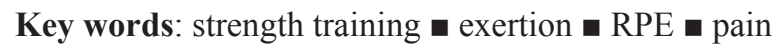

\section{INTRODUCTION}

Rating of perceived exertion (RPE) scales have been applied in a wide range of physical activities and exercise modalities in attempt to identify the degree of effort put forth by individuals during their performance. Despite being originally developed for use with aerobic type exercise, the use of RPE scales within resistance training (RT) has become more widespread. ${ }^{1}$ Though commonly employed as a tool to measure perceived effort the first studies regarding psychophysical scaling considered perceptions of heaviness in relation to the load employed during activities. ${ }^{2}$ Subsequently the application of RPE scales of a variety of kinds (Borg 15 point Scale, Borg Category 10 Ratio, or the OMNI Resistance Exercise Scale) have been examined for construct validity against the external load employed. Indeed many of these scales use terminology relating to load and the OMNI, for example uses images of increasing load as descriptors.
Numerous studies have revealed that participants report increasingly higher RPE values with increasing load/torque demands $^{3-8}$ even when matched for volume-load (repetitions $\mathrm{x}$ load $)^{9-11}$. In addition a number of studies have reported a relationship between load, RPE and physiological measures such as: electromyography amplitude $\mathrm{e}^{3,6,8,12}$, heart rate $^{5}$, and blood lactate $^{12,13}$. This body of research has served to further reinforce the potential value of applying RPE to RT with a focus upon the prescription, control and monitoring of load..$^{14,15}$

However, a concern with the current body of research is a lack of consideration for the role of fatigue and proximity to momentary failure (MF); in essence the point at which despite the greatest effort the trainee is unable to meet and overcome the demands of the exercise. Research has consistently supported that higher RPE values are reported as participants achieve close proximity to MF by performing an increasing number of repetitions or time under load. ${ }^{4,16-18}$ Also when vol-

Received September 26, 2016; accepted December 12, 2016

From the Centre for Health, Exercise and Sport Science, Southampton Solent University, East Park Terrace, Southampton, UK (J.S., J.F.), and Abstract Bodyworks, Newbury, UK (S.M., P.M.) Communicated by Takashi Abe, PhD

Correspondence to James Steele, Centre for Health, Exercise and Sport Science, Southampton Solent University, East Park Terrace, Southampton, Hampshire, UK, SO14 0YN, Email: james.steele@solent.ac.uk

Journal of Trainology 2017;6:1-8 @2012 The Active Aging Research Center http://trainology.org/ 
ume-load is matched the higher load conditions often are performed closer to MF thus producing higher RPE ${ }^{1}$ suggesting that RPE may be related to fatigue and the proximity to MF. Since it is perception of effort that we are trying measure when we use RPE it is evident that as fatigue increases so the RPE values reported increase. With this understanding it would perhaps be most appropriate to anchor the intensity of perceived effort as being maximal at the point of $\mathrm{MF}^{19}$, this being partly due to the consideration of perceived effort being relative to the ability to meet the demands of an exercise or activity. In support, Allman \& Rice ${ }^{20}$ reported that, after anchoring RPE to the sensation of effort during a maximal voluntary contraction (MVC), participants performing repeated isometric contractions at $60 \% \mathrm{MVC}$ to the point of MF consistently reported maximal RPE values upon reaching this point. Indeed to understand submaximal effort with this conceptualisation some studies have attempted to develop scales that assess effort during RT relative to MF. ${ }^{21,22}$ However, a further consideration in this conceptualisation should also be the apparent difficulty people experience in differentiating between perceptions of effort and discomfort.

A recent review ${ }^{23}$ has discussed the differentiation between what is termed effort, defined as "the amount of mental or physical energy being given to a task" (which we would note is a relative perception), and exertion defined as "the amount of heaviness and strain experienced in physical work". The authors of this review noted that both terms are often used interchangeably and in certain languages translate as synonyms. As such we suggest discomfort which has previously been used to describe the physiological and unpleasant sensations associated with exercise. ${ }^{24}$ Thus for practical purposes here we have opted to use the term discomfort as opposed to exertion.

This differentiation between perceptions of effort and discomfort has been highlighted recently as important ${ }^{23,25}$ particularly within $\mathrm{RT}^{19}$. A number of studies ${ }^{20,26-30}$ have reported that participants exercised to MF with verbal encouragement to ensure adequate motivation and effort where RPE was measured. In this case, each trial, irrespective of exercise, load, or training status would be expected to result in a maximal value for perceived effort since people were exercising to MF. Though those studies which have compared training to MF with training not to MF show that RPE for the active muscle is indeed higher when training to $\mathrm{MF}^{26,27}$, with one exception ${ }^{20}$, the RPE values reported by participants were not maximal in any of the studies cited above. Thus we can only assume that either; participants did not exercise to MF, or the participants were unclear as to how to report their perception of effort. Increasing ratings of effort, despite conditions being controlled by training to supposed MF, were however given with lower load for lower body exercise ${ }^{29}$, as set volume's increased ${ }^{30}$, with increased volume-load ${ }^{28}$, and with increased work rate re,27 $^{26}$ supporting that participants may have expressed their feelings of increasing discomfort ${ }^{19,25}$.

Some studies have attempted to differentiate between effort and discomfort during RT and have found that, though participants appear able to report different values for each, there is a similar pattern for both responses. Studies by Hollander et $\mathrm{al}^{31,32}$ found that, though RPE is typically reported as being higher than discomfort (the authors used the term pain) for a range of RT conditions (different loads and contraction types), both respond in a similar fashion. Studies using blood flow restriction based RT have shown similar findings ${ }^{33,34}$, though it should perhaps be noted that this unique form of exercise typically produces greater discomfort post completion (i.e. during the rest periods). This relationship between the two may be inherent, however, it has been evidenced that the perception of effort is independent from afferent feedback mechanisms. ${ }^{24}$ This would seem to disagree with observations in the studies discussed of higher RPEs under conditions known anecdotally to induce higher feelings of discomfort. Smirnaul ${ }^{25}$ (p. 309) offered a practical example to highlight the relationship between effort and discomfort if the two are appropriately differentiated: "A short maximal voluntary contraction for leg extension, for example, will by nature induce a maximal sense of effort while, initially, other unpleasant sensations will probably be modest. Repeating this maximal contraction several times, however, will increase these unpleasant sensations continuously, whereas the sense of effort will be always the same (i.e. maximal)." It is not clear from the methods used by the authors of the studies highlighted above whether the descriptions of the scales used explicitly asked participants to differentiate between the effort and discomfort. Though these studies may have utilised the instructions provided within the original Borg Category 10 Ratio exertion and pain scales, the descriptors used for exertion in these scripts could be interpreted by participants similarly (i.e. the exertion scale asks participants to report based upon the 'strain' in the muscles and 'feelings of breathlessness or aches in the chest' all of which may or may not be related to perceived effort). It is therefore possible that participants were either consciously or unconsciously anchoring their effort and discomfort responses upon one another.

It has been concluded in recent reviews that training to MF may be optimal for improving strength ${ }^{35}$ and hypertrophy ${ }^{36}$. However, more current work has indicated that there may be a dose-response relationship to the role of intensity of effort in RT that is not yet fully understood. For example, in an untrained population it was reported that strength gains may be similar when RT performed to MF or not to MF is matched for load and volume with the authors speculating that the increasing RPE resulting from the cumulative fatigue of the not to MF protocol may have resulted in the similar improvements. ${ }^{37}$ In contrast, another study examining well trained participants found that having participants train to the point where they predicted MF on their subsequent repetition resulted in no significant improvements in strength or hypertrophy whereas training to MF did. ${ }^{38}$ Though in this study there were greater adaptations when participants trained to a maximal effort (i.e. $\mathrm{MF}$ ), the degree of perceived effort when training to the point where MF is predicted on the next repetition was unknown. As such, clearly there is some submaximal intensity of effort insufficient to stimulate optimal adaptation, however, the specific nature of the dose-response relationship between submax- 
imal efforts and adaptations is currently unclear.

The anchoring of maximal effort as being synonymous with MF in RT provides a point from which to examine the role of differing intensities of perceived effort during submaximal efforts. This might permit further understanding of the doseresponse role of perceived effort during RT. There may be a submaximal effort threshold whereby adaptations are optimised which would be of considerable value in instances where training to MF is not advised or contraindicated. However, scales permitting appropriate differentiation between effort and discomfort may also be an essential component of research endeavours in this regard. Further, research examining RT under different manipulations of variables whilst differentiating perceived effort and discomfort might provide valuable practical information regarding exercise tolerance. For example, a protocol that maximises effort yet produces a relatively low perceive discomfort may impact upon adherence. The aim of this study therefore was to evaluate the use of two scales for perception of effort and discomfort designed to permit appropriate differentiation between the two.

\section{METHODS}

\section{Experimental Approach to the Problem}

Two novel scales with accompanying scripts were designed to permit differentiation between perceptions of effort and discomfort during RT. In order to determine whether participants were able to differentiate adequately between these two constructs, in addition to whether the scales were reliable, participants in this study underwent 3 RT trials over a period of 3 weeks consisting of full body workouts training to a self-determined 6 repetition maximum. During each trial, after completion of each exercise participants were asked to provide a rating for RPE-E and RPE-D. In addition, and before participants provided their ratings, the trainer recorded their assessment of the participants RPE-E in order to examine the reliability and validity of their assessments.

\section{Participants}

Seventeen participants (males $n=6$, females $n=11$, age $63 \pm 16$ years) were recruited from the existing membership pool of a private UK based exercise facility. Participants were required to have no medical condition for which RT would be contraindicated to participate and all had been engaged in resistance training $1-2 \mathrm{~d} \cdot \mathrm{wk}^{-1}$ for $\geq 3$ months prior to testing using the same training protocol examined in the present study. Informed consent was provided by all participants and the study was approved by the relevant ethics committee at the author's institution.

\section{Procedures}

Equipment and testing

RT was performed using MedX (MedX Corporation, FL, USA) chest press, torso arm (pull down), seated row, overhead press, and leg press resistance machines. Two distinct 11 point scales $(0-10)$ were produced for the present study for assessing perceptions of effort (RPE-E; figure 1) and perceptions of discomfort (RPE-D; figure 2) separately during RT. A script (cop- ies of the scripts are available from the contact author upon request) was developed to accompany these scales to explain to participants to differentiate between what the scales were asking them to report and what the anchors for the scales were (see supplemental digital content). All participants were required to read the script and to confirm their understanding prior to beginning testing.

The RPE-E scale was anchored as follows:

"The scale begins at 0 which is defined as no physical exertion is taking place. This can be likened to your perception of effort sitting on a machine but remaining motionless. The scale ends at 10 which is described as the maximum perceivable effort. This can be likened to your perception of effort when, despite putting forth as much exertion as you can, you cannot physically complete the activity being attempted."

\section{HOW HARD DO YOU THINK YOU'RE WORKING?}

\begin{tabular}{|l|r|}
\hline 0 & NO EXERTION \\
\hline 1 & EXTREMELY EASY \\
\hline 2 & EASY \\
\hline 3 & \\
\hline 4 & \\
\hline 5 & SOMEWHAT HARD \\
\hline 6 & \\
\hline 7 & HARD \\
\hline 8 & VERY HARD \\
\hline 9 & MAXIMAL EFFORT \\
\hline 10 & \\
\hline
\end{tabular}

Figure 1 RPE-E scale for assessing perceived effort

\section{HOW MUCH DISCOMFORT DO YOU FEEL?}

\begin{tabular}{|l|r|}
\hline 0 & NO DISCOMFORT \\
\hline 1 & MINOR DISCOMFORT \\
\hline 2 & \\
\hline 3 & MILD DISCOMFORT \\
\hline 4 & \\
\hline 5 & MODERATE DISCOMFORT \\
\hline 6 & SEVERE DISCOMFORT \\
\hline 7 & \\
\hline 8 & MAXIMAL DISCOMFORT \\
\hline 9 & VERY SEVERE DISCOMFORT \\
\hline 10 & \\
\hline
\end{tabular}

Figure 2 RPE-D scale for assessing perceived discomfort 
The RPE-D scale was anchored as follows:

"The scale begins at 0 which is described as no perceived discomfort. This can be likened to a perception of discomfort at a time where you feel no noticeable sensations relating to physical activity. The scale ends at 10 which is described as the maximum perceivable discomfort. This can be likened to a perception of discomfort where you could not imagine the sensations relating to physical activity being any more intense."

Based on the way in which the term effort was defined in the present study it was considered difficult to experimentally determine the degree of construct validity for the RPE-E scale produced. Effort was defined relative to a person's perceived ability to meet the demands of an activity or exercise. Without a participant continuing to the point at which they can no longer meet those demands (which from performance to performance may vary) it is impossible to know whether a person's given rating of effort matches the actual relative degree of effort required at a given moment during submaximal exercise performance which could be defined as:

$$
\left(\frac{A}{D}\right) \times 100
$$

Where,

Equation 1.

$A=$ Current ability to meet demands of the activity or exercise

And,

$$
D=\text { The demands of the activity or exercise }
$$

Therefore, the RPE-E scale developed and used here could be considered similarly to a visual analogue scale anchored at either end. Though discouraged in development of other RPE scales, the use of a top anchor as used here was appropriate as it was anchored based upon the definition of effort used and what this would mean during RT when maximal (i.e. a person reaching $\mathrm{MF}$ ). As the function that would mathematically describe the relationship of perceived effort under the present definition is unknown, equidistant descriptors were chosen to assist participants in providing ratings of effort. It was considered these, along with the script provided, were adequate in terms of content validity; that is they allowed the scale to measure perceived effort in the way defined here and that both the investigators and participants understood this. As it was of interest to examine participant's ability to differentiate between perceived effort and discomfort in the present study the RPE-D scale developed for discomfort was similar in appearance and description. This was to examine whether participants could conceptually differentiate the two perceptions despite measuring them on similar 11 point scales.

Participants were instructed to report their RPE-E and RPE-D verbally and in that order immediately (so that anchoring of RPE-E to RPE-D was not affected by priming effects) following completion of each exercise. In addition, and prior to the participants report, the trainer recorded their assessment of the participants RPE-E in order to examine the validity of trainer ratings compared with participants. This was also in order to determine, similarly to Borg's RPE and CR10 scales ${ }^{39}$, whether content validity was adequate. Trainers ratings were based upon their prior experiences with training persons in the manner described below and were based around observations including an increased breathing frequency, shaking of limbs, facial expressions (grimacing), reduction in repetition duration, attempts to change posture (exercise form), and whether the participant appeared to achieve MF. This was not revealed to the participant. For the first trial, in a sub set of participants $(n=4)$, an additional trainer was available and observed the trials and also recorded an assessment of participants RPE-E. The same trainers delivered the RT sessions and provided ratings for each participant for each trial.

\section{Resistance Training Protocol}

Participants underwent 3 RT trials over a period of 3 weeks (at least 1 week between trials) consisting of 5 compound exercises (chest press, pull down, seated row, overhead press and leg press). Order of exercises was the same for each trial and participants began each successive exercise following $~ 30$ seconds of rest. Each exercise was completed with a load that allowed the participants to train to a self-determined 6RM (meaning they determined inability to complete further repetitions if attempted i.e. they predicted momentary failure on the next repetition) through a full range of motion using a repetition duration of 10 seconds concentric and 10 seconds eccentric. This equated to a total repetition duration and time under load of $\sim 120$ seconds. The trainer monitored participants repetition duration throughout each exercise using a stopwatch and advised participants to either speed up or slow down as appropriate to maintain this repetition duration within a margin of 2 seconds error (i.e., 8-12 s concentric: $8-12$ s eccentric). The trainers throughout this intervention encouraged very strict form during exercise; for example, controlled and continuous breathing frequency (without a valsalva manoeuvre), attempting to keep muscles which are not the target of the exercise as relaxed as possible and ensuring the ability to maintain interrepetition consistency to the prescribed repetition duration within the set. A self-determined RM was chosen as the repetition cessation criteria in order to examine the use of the RPE-E and RPE-D scales where participants might not be achieving MF (i.e. during submaximal effort RT), though in some instances during trials participants did reach MF (in all cases where MF was reached a rating of 10 for RPE-E was provided by participants).

\section{Statistical Analysis}

In order to examine whether participants were able to differentiate between RPE-E and RPE-D we also examined Spearman's Rank Correlations between the two variables for each exercise and for all combined data across the 3 trials. Intra-rater absolute standard error of measurement (SEM) was calculated for trainee RPE-E, trainee RPE-D, and trainer RPEE. In addition, in the sub-set of participants receiving 2 trainer RPE-E assessments $(n=4)$, inter-rater absolute SEM was calculated. SEM was also used to examine the agreement between trainee RPE-E and trainer RPE-E. SEM was used to reflect the variation of the measures upon repeated testing and 
amongst observers. First the standard deviation across trials (intra- and inter-rater) for all participants was determined, this was then squared and the absolute SEM calculated as the following equation:

$$
\text { Absolute SEM }=\sqrt{\frac{\sum \sigma_{i}^{2}}{2 n}}
$$

Where:

Equation 2.

$\Sigma \sigma^{2}=$ summation of standard deviations squared

$n=$ number of participants measured

$i=$ number of standard deviations

In addition, validity of trainer RPE-E was also examined as SEM between trainer and trainee RPE-E. Calculations were performed using Microsoft Office Excel 2013 (Microsoft Corporation, Redmond, WA, USA) and statistical analysis performed using IBM SPSS Statistics for Windows (version 20; IBM Corp, Portsmouth, Hampshire, UK) and $p \leq 0.05$ set as the limit for statistical significance.

\section{RESULTS}

Though significant, Spearman's Rank correlations between RPE-E and RPE-D were weak and ranged from $r=0.373$ to 0.492 across the individual exercises (all $p<0.01$ ) and correla-

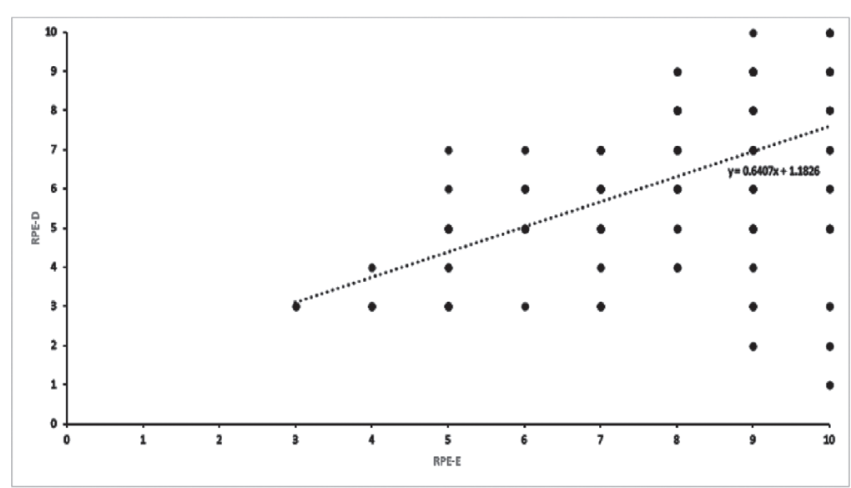

Figure 3 Scatter plot of RPE-E and RPE-D for combined data tion for the combined data was also weak $(r=0.479 ; p<$ 0.0001; see figure 3).Intra-rater SEMs for trainee RPE-E ranged from 0.64 to $0.85 \mathrm{pts}$, trainee RPE-D ranged from 0.60 to $1.00 \mathrm{pts}$, and trainer RPE-E ranged from 0.56 to 0.71 . Interrater SEMs for trainer RPE-E ranged 0.25 to 0.66 . Results suggest that the reliability for both trainee measures of RPE-E and RPE-D, in addition to trainer RPE-E is acceptable. SEMs for agreement between trainer and trainee RPE-E ranged from 1.03 to 1.25 . RPE-E and RPE-D for each trial, and individual SEMs including for trainer-trainee agreement for each exercise tested are presented in table 1. Inter-rater SEMs for trainer RPE-E ranged from 0.25 to 0.66 indicating good agreement and these are presented in table 2 .

\section{DISCUSSION}

The present study sought to examine the use of two scales for perception of effort (RPE-E) and discomfort (RPE-D) designed to permit appropriate differentiation between the two constructs. Spearman's Rank correlations revealed a significant but weak correlation between the two measures for all exercises tested suggesting that participants were able to appropriately differentiate between the two constructs being measured. Further, SEMs for trainee RPE-E and RPE-D in addition to trainer RPE-E showed acceptable reliability suggesting that these scales could be reliably used to monitor and investigate the impact of sub-maximal perceived effort within RT.

Table 2 Inter-rater SEMs for Trainer RPE-E for each exercise.

\begin{tabular}{cccc}
\hline & \multicolumn{3}{c}{ Trainer RPE-E } \\
\hline & Rater 1 & Rater 2 & SEM \\
\hline Chest Press & $7.50 \pm 1.29$ & $7.25 \pm 0.96$ & 0.25 \\
\hline Row & $6.25 \pm 1.50$ & $6.75 \pm 1.50$ & 0.50 \\
\hline Pulldown & $7.25 \pm 1.50$ & $7.00 \pm 0.00$ & 0.66 \\
\hline Overhead Press & $8.00 \pm 0.82$ & $8.75 \pm 0.50$ & 0.56 \\
\hline Leg Press & $7.25 \pm 0.96$ & $7.50 \pm 1.00$ & 0.56 \\
\hline
\end{tabular}

Table 1 Trainee RPE-E, RPE-D, trainer RPE-E for each trial and exercise and SEMs including for trainer-trainee RPE-E agreement.

\begin{tabular}{|c|c|c|c|c|c|c|c|c|c|c|c|c|c|}
\hline & \multicolumn{4}{|c|}{ Trainee RPE-E } & \multicolumn{5}{|c|}{ Trainee RPE-D } & \multicolumn{3}{|c|}{ Trainer RPE-E } & \multirow{2}{*}{$\begin{array}{l}\text { Trainer } \\
\text { Trainee } \\
\text { RPE-E } \\
\text { SEM }\end{array}$} \\
\hline & Trial 1 & Trial 2 & Trial 3 & SEM & Trial 1 & Trial 2 & Trial 3 & SEM & Trial 1 & Trial 2 & Trial 3 & SEM & \\
\hline Chest Press & $7.53 \pm 1.55$ & $7.35 \pm 1.73$ & $7.65 \pm 1.58$ & 0.64 & $5.18 \pm 1.81$ & $5.59 \pm 2.09$ & $6.06 \pm 1.92$ & 0.60 & $6.94 \pm 1.20$ & $7.41 \pm 1.23$ & $7.82 \pm 1.01$ & 0.67 & 1.25 \\
\hline Row & $7.41 \pm 1.66$ & $7.29 \pm 1.53$ & $7.82 \pm 1.55$ & 0.82 & $5.00 \pm 2.18$ & $5.59 \pm 2.12$ & $6.47 \pm 1.87$ & 0.87 & $7.00 \pm 1.80$ & $7.41 \pm 1.18$ & $7.71 \pm 1.21$ & 0.63 & 1.15 \\
\hline Pulldown & $7.94 \pm 2.08$ & $7.29 \pm 1.76$ & $8.18 \pm 1.74$ & 0.65 & $5.94 \pm 2.41$ & $6.00 \pm 2.34$ & $6.94 \pm 2.08$ & 0.72 & $7.59 \pm 2.06$ & $7.59 \pm 1.37$ & $8.06 \pm 1.71$ & 0.57 & 1.07 \\
\hline $\begin{array}{l}\text { Overhead } \\
\text { Press }\end{array}$ & $8.35 \pm 1.66$ & $7.94 \pm 1.43$ & $8.65 \pm 1.62$ & 0.71 & $6.88 \pm 2.00$ & $6.59 \pm 2.35$ & $7.53 \pm 1.70$ & 1.01 & $8.59 \pm 1.46$ & $8.12 \pm 1.27$ & $8.65 \pm 1.32$ & 0.71 & 1.11 \\
\hline Leg Press & $7.82 \pm 1.67$ & $7.71 \pm 1.45$ & $7.88 \pm 1.50$ & 0.85 & $6.00 \pm 2.00$ & $6.12 \pm 2.15$ & $6.71 \pm 2.08$ & 0.86 & $7.88 \pm 1.50$ & $7.65 \pm 1.00$ & $7.82 \pm 1.38$ & 0.68 & 1.03 \\
\hline
\end{tabular}


As noted, differentiation between perceptions of effort and discomfort are important to consider if an appropriate understanding of the role of perceived effort during exercise is to be obtained. ${ }^{19,23-25}$ Certain manipulations of RT variables appear to have the potential to impact upon RPE in unexpected ways and thus may indicate a conflation of perceived effort and discomfort by participants. ${ }^{26-30}$ Indeed, even where studies have attempted to differentiate the two, strong relationships in their responses manifest both during traditional RT and blood flow restriction based $\mathrm{RT}^{31-34}$. This may be potentially be due to these studies in some cases using high effort RT conditions known also to anecdotally produce high levels of discomfort (e.g. blood flow restriction or relatively low/moderate loads $[65-80 \% 1 \mathrm{RM}])$. However, Robertson et al. ${ }^{40}$ found that in children there was no particular pattern of relationship between 'muscle hurt' and RPE favouring any particular loading conditions $(30 \%, 50 \%$, or $70 \%$ of $1 \mathrm{RM})$. Prior studies have not clarified whether, though participants were asked to provide values for the two constructs, they were explicitly instructed to differentiate between the two. Within the present study we developed scales and an accompanying script explicitly instructing participants to consider the two as separate constructs that could be differentiated from one another. It was explained that it was possible for participants to perceive a high degree of effort and relatively little discomfort and also vice-versa. Though our results indicated that a relationship did exist between the RPE-E and RPE-D, in comparison to prior research this was relatively weak thus suggesting that the use of an appropriate script with the two scales for effort and discomfort allowed participants to appropriately differentiate between them. Indeed, figure 3 highlights that at higher RPE-E values this differentiation between the two was even more apparent. This clear ability to differentiate between the two constructs supports the content validity of the scales used. In addition, the acceptable agreement between both trainer and trainee RPE-E ratings further suggested that adequate content validity of the scale was met.

The SEMs reported in the present study for trainee RPE-E and RPE-D suggested acceptable reliability. The scales therefore present potential applications from both a research and practical perspective. Prior research examining the role of effort during RT has been required primarily to consider it from a dichotomous perspective; to MF (maximal) or not to MF (sub-maximal). Though research suggests that training to MF may be optimal for a range of outcomes ${ }^{35,36}$ there may in fact be a sub-maximal effort threshold whereby these adaptations are optimised. There might also be instances where training to MF is not advised or contraindicated and so knowing what sub-maximal efforts will still optimise outcomes has practical value. Relatively little is understood about the doseresponse nature of sub-maximal effort during RT. As noted, in untrained persons it might be possible to optimise strength adaptations despite not training to $\mathrm{MF}$ as the cumulative fatigue incurred across multiple sets not to MF increases the effort required. ${ }^{37}$ Contrastingly, when trained persons attempt to predict themselves as being 1 repetition away from MF adaptations are blunted. ${ }^{38}$ However, no research has attempted to examine the dose-response relationship of a range of submaximal perceived efforts. The SEMs reported here suggest that these scales could be implemented in research examining training interventions where participants are asked to work to a particular sub-maximal effort rating across sufficiently differentiated ratings (e.g. 2, 4, 6, 8, and 10 based upon our SEMs). Volume-load could be controlled for between groups by having participants perform each set until a specified RPE-E was met and continue performing sets with sufficient inter-set rest until a specified total number of repetitions were completed.

The results of the present study should be considered in light of possible limitations. The mean age of the participants was age $63 \pm 16$ years and that 11 of the 17 participants were female thus the present results should perhaps be considered as applicable predominantly to older female populations. Although patterns of RPE are similar between populations, some research suggests that older adults ( $\sim 75$ years) might under-estimate RPE compared with young adults ${ }^{8}$ this difference diminishes as RT nears $\mathrm{MF}^{20}$. Further, most research suggests that any gender differences in RPE are minor ${ }^{8,17,41}$ and both these and age differences are not particularly meaning$\mathrm{ful}^{4}$. Also, most research relating to RPE during RT has used participants with $\geq 3$ months prior regular RT experience similar to the sample in the present study. When examined with reference to increasing load prior work is contrasting as to whether differences in RPE exist between trained and untrained persons. ${ }^{42,43}$ However, again it seems that any difference that might exist becomes less meaningful as trainees approach $\mathrm{MF}^{18}$. This suggests that the scales examined here might be suitable for use in young and old, male and female, and trained and untrained populations, when RT is performed near to or at MF. However, further research should directly examine their application in other populations to confirm this. Lastly, though we opted to use a set end-point which would permit a range of potential submaximal efforts to be investigated (i.e. a self-determined 6RM), the fact that we only used this repetition range/relative load could be considered a limitation. It may be that the scales are less sensitive to enabling participants to differentiate between effort and discomfort under conditions where the two may be more closely related (e.g. low loads performed to MF). Thus future work might look to investigate these scales across a range of loads and repetition ranges in addition to under alternative conditions (e.g. blood flow restriction).

Though the scales examined here present value for further examining the dose-response nature of sub-maximal effort during RT, it should be noted that they also present a number of potential practical applications. Monitoring of RT by trainers and coaches using subjective measures is becoming more widespread and recommended ${ }^{1}$. Indeed such subjective measures as RPE might be useful in ensuring that athletes and other persons avoid overtraining. ${ }^{44}$ The prior consideration of effort in relation to MF as being dichotomous (to MF or not to $\mathrm{MF}$ ) presents twofold practical issues. If trainees perform RT to $\mathrm{MF}$ in order to control for effort from set to set, exercise to exercise, session to session etc. then there may be the potential for overtraining to occur. ${ }^{45}$ Conversely if trainees are not train- 
ing to MF (either using a prescribed repetition number or trying to predict proximity to MF) then it may not be clear the degree of relative effort they are in fact putting forth. Use of the scales examined here however might permit appropriate monitoring of training when performing RT not to MF. Indeed, trainee ratings of RPE-E here showed good reliability from session to session suggesting that sub-maximal RPE-Es might be prescribed during training sessions and that progression might be made around them. Further, it appears that trainers are also able to rate trainees RPE-E with good reliability and that there is high agreement between trainers. Though not as high as the inter- or intra-rater agreements examined for reliability (SEMs ranging 0.25 to 0.85 ) we also found that trainer ratings of RPE-E had reasonable agreement with trainee ratings of RPE-E (SEMs ranging 1.03 to 1.25 ) suggesting that appropriately skilled trainers can provide a valid estimate of trainee effort during RT. This may be of practical use to coaches to monitor training were it might be inappropriate or inconvenient to ask the trainee during RT to provide an RPE-E rating themselves.

In conclusion, the two scales and script examined here for perceived effort (RPE-E) and discomfort (RPE-D) enabled trainees to appropriately differentiate between the two and provide reliable ratings. These results suggest that the scales might be used in research examining the dose-response nature of perceived effort independently of perceived discomfort upon outcomes related to RT. Further, they might also be employed in practical settings to monitor sub-maximal effort during RT and as trainers showed good validity and reliability in assessing trainee RPE-E they may be able to use such information in programming.

\section{COMPLIANCE}

The authors received no funding in support of this project.

\section{REFERENCES}

1. Scott BR, Duthie GM, Thornton HR, Dascombe BJ. Training monitoring for resistance exercise: theory and applications. Sports Med, Epub ahead of print 2016

2. Stevens SS. On the psychophysical law. Psychol Rev 1957;64:153-181.

3. Andersen LL, Andersen CH, Mortensen OS et al. Muscle activation and perceived loading during rehabilitation exercises: Comparison of dumbbells and elastic resistance. Phys Ther 2010;90:538-549.

4. Buckley JP, Borg GAV. Borg's scales in strength training; from theory to practice in young and older adults. Appl Physiol Nutr Metab 2011;36:682692.

5. Colado JC, Garcia-Masso X, Triplett TN et al. Concurrent validation of the OMNI-Resistance exercise scale of perceived exertion with thera-band resistance bands. J Strength Cond Res 2012;26:3018-3024.

6. Hollander DB, Worley JR, Asoodeh M et al. A comparison of resistance exercise perceived exertion and muscle activation at varied submaximal durations, loads, and muscle actions. J Strength Cond Res, Epub ahead of print 2015.

7. Lins-Filho ODL, Robertson RJ, Farah BQ et al. Effects of exercise intensity on a rating of perceived exertion during a multiple-set resistance exercise session. J Strength Cond Res 2012;26:466-472.

8. Pincivero DM. Older adults underestimate RPE and knee extensor torque as compared with young adults. Med Sci Sports Exerc 2011;43:171-180.

9. Gearhart RE, Goss FL, Lagally KM et al. Standardized scaling procedures for rating perceived exertion during resistance exercise. J Strength Cond
Res 2001;15:320-325.

10. Gearhart RF, Goss FL, Lagally KM et al. Ratings of perceived exertion in active muscle during high-intensity and low-intensity resistance exercise. $J$ Strength Cond Res 2002;16:87-91.

11. Lagally KM, Robertson RJ, Gallagher KI et al. Ratings of perceived exertion during low- and high-intensity resistance exercise by young adults. Percept Mot Skills 2002;94:723-731.

12. Lagally KM, Robertson RJ, Gallagher KI et al. Perceived exertion, electromyography, and blood lactate during acute bouts of resistance exercise. Med Sci Sports Exerc 2002;34:552-559.

13. Robertson RJ, Goss FL, Rutkowski J et al. Concurrent validation of the OMNI perceived exertion scale for resistance exercise. Med Sci Sports Exerc 2003;35:333-341.

14. Gearhart RF, Lagally KM, Riechman SE et al. Strength tracking using the OMNI resistance exercise scale in older men and women. J Strength Cond Res 2009;23:1011-1015.

15. Gearhart RF, Lagally KM, Riechman SE et al. RPE at relative intensities after 12 weeks of resistance-exercise training by older adults. Percept Mot Skills 2008;106:893-903.

16. Pincivero DM, Gear WS. Quadriceps activation and perceived exertion during a high intensity, steady state contraction to failure. Muscle Nerve 2000;23:514-520.

17. Pincivero DM, Coelho AJ, Campy RM. Gender differences in perceived exertion during fatiguing knee extensions. Med Sci Sports Exerc 2004; 36:109-117.

18. Testa M, Noakes TD, Desgorces F. Training state improves the relationship between rating of perceived exertion and relative exercise volume during resistance exercises. J Strength Cond Res 2012;26:2990-2996.

19. Steele J. Intensity; in-ten-si-ty; noun. 1. Often used ambiguously within resistance training. 2. Is it time to drop the term altogether? Br J Sports Med 2014;48:1586-1588.

20. Allman BL, Rice CL. Perceived exertion is elevated in old age during an isometric fatigue task. Eur J Appl Physiol 2003;89:191-197.

21. Hackett DA, Johnson NA, Halaki M et al. A novel scale to assess resistance-exercise effort. J Sports Sci 2013;30:1405-1413.

22. Zourdos MC, Klemp A, Dolan C et al. Novel resistance training-specific rating of perceived exertion scale measuring repetitions in reserve. $J$ Strength Cond Res 2015;30:267-275.

23. Abbiss CR, Peiffer JJ, Meeusen R et al. Role of ratings of perceived exertion during self-paced exercise: What are we actually measuring? Sports Med 2015;45: 1235-1243.

24. Marcora S. Perception of effort during exercise is independent of afferent feedback from skeletal muscles, heart, and lungs. J Appl Physiol 2009;106: 2060-2062.

25. Smirnaul Bde P. Sense of effort and other unpleasant sensations during exercise: clarifying concepts and mechanisms. Br J Sports Med 2012;46: 308-311.

26. Hiscock DJ, Dawson B, Peeling P. Perceived exertion responses to changing resistance training programming variables. J Strength Cond Res 2015;29:1564-1569.

27. Hiscock DJ, Dawson B, Donnelly CJ et al. Muscle activation, blood lactate, and perceived exertion responses to changing resistance training programming variables. Eur J Sports Sci, Epub ahead of print 2015

28. Pritchett RC, Green JM, Wickwire PJ et al. Acute and session RPE responses during resistance training: Bouts to failure at $60 \%$ and $90 \%$ of 1RM. SAJSM 2009;21:23-26.

29. Shimano T, Kraemer WJ, Spiering BA et al. Relationship between the number of repetitions and selected percentages of one repetition maximum in free weight exercises in trained and untrained men. $J$ Strength Cond Res 2006;20:819-823.

30. Silva VL, Azevedo AP, Cordeiro JP et al. Effects of exercise intensity on perceived exertion during multiple sets of bench press to volitional failure. J Trainol 2014;3:41-46.

31. Hollander DB, Duran RJ, Trynicki JL et al. RPE, pain, and physiological adjustment to concentric and eccentric contractions. Med Sci Sports Exerc 
2003;35:1017-1025.

32. Hollander DB, Kilpatrick MW, Ramadan ZG et al. Load rather than contraction type influences rate of perceived exertion and pain. J Strength Cond Res 2008;22:1184-1193.

33. Loenneke JP, Balapur A, Thrower AD et al. The perceptual responses to occluded exercise. Int J Sports Med 2011;32:181-184.

34. Loenneke JP, Kim D, Fahs CA et al. The effects of resistance exercise with and without different degree of blood-flow restrictions on perceptual responses. J Sports Sci 2015;33:1472-1479.

35. Fisher J, Steele J, Bruce-Low S et al. Evidence-based resistance training recommendations. Med Sport 2011;15:147-162.

36. Fisher J, Steele J, Smith D. Evidence-based resistance training recommendations for muscular hypertrophy. Med Sport 2013;17: 217-235.

37. Fisher JP, Blossom D, Steele J. A comparison of volume-equated knee extension to failure, or not to failure, upon rating of perceived exertion and strength adaptations. Appl Physiol Nutr Metab 2015;41:168-174.

38. Giessing J, Fisher J, Steele J et al. The effects of low volume resistance training with and without advanced techniques in trained participants. $J$ Sports Med Phys Fitness 2016;56: 249-258.
39. Borg G. Borg's Perceived Exertion and Pain Scales. Champaign, IL: Human Kinetics, 1998

40. Robertson RJ, Goss FL, Aaron DJ et al. Concurrent muscle hurt and perceived exertion of children during resistance exercise. Med Sci Sports Exerc 2009;41:1146-1154.

41. Springer BK, Pincivero DM. Differences in ratings of perceived exertion between the sexes during single-joint and whole-body exercise. $J$ Sports Sci 2010;28:75-82.

42. Lagally KM, McCaw ST, Young GT et al. Ratings of perceived exertion and muscle activity during the bench press exercise in recreational and novice lifters. $J$ Strength Cond Res 2004;18:359-364.

43. Tiggemann CL, Korzenowski AL, Brentano MA et al. Perceived exertion in different strength exercise loads in sedentary, active, and trained adults. J Strength Cond Res 2010;24:2032-2041.

44. Foster C. Monitoring training in athletes with reference to over-training syndrome. Med Sci Sports Exerc, 1998;30:1164-1168.

45. Willardson J. The application of training to failure in periodized multiple set resistance exercise programs. J Strength Cond Res 2007;21:628-631. 